\title{
Non-invasive fractional flow reserve estimation with coronary computed tomography angiography
}

\begin{abstract}
Coronary computed tomography calcium score and angiography has emerged as a powerful tool in the investigation of patients with chest pain. Coronary computed tomography calcium score provides both diagnostic as well as prognostic information but in the presence of intermediate coronary lesions, neither coronary CT calcium score nor CT angiography is able to determine whether such a lesion is functionally significant. Fractional flow reserve coronary $\mathrm{CT}$ angiography has emerged as a novel, non-invasive technique for the assessment of intermediate atherosclerotic coronary artery lesions.
\end{abstract}

Keywords: coronary artery disease, fractional flow reserve, coronary computed tomography angiography
Volume 2 Issue 2 - 2016

\author{
Bassey Ussen,' Nikunj Shah,' Nicholas \\ Curzen, ${ }^{2}$ Michael Mahmoudi? ${ }^{1,2}$ \\ 'Ashford \& St Peter's Hospitals NHS Foundation Trust, UK \\ 'University Hospital Southampton, NHS Foundation Trust, UK
}

Correspondence: Bassey Ussen, Ashford \& St Peter's

Hospitals NHS Foundation Trust,

Email bassey.ussen@asph.nhs.uk

Received: January 18, 2016 | Published: February 12, 2016
Abbreviations: CAD, coronary artery disease; FFR, fractional flow reserve; CTA, coronary computed tomography angiography

\section{Introduction}

Coronary artery disease (CAD) remains a significant cause of morbidity and mortality worldwide. ${ }^{1}$ Myocardial ischemia results when myocardial blood flow is insufficient to meet demand; this causes symptoms and affects patient outcome. ${ }^{2}$ According to current guidelines for the management of stable $\mathrm{CAD}$, revascularisation should be guided by the presence of myocardial ischaemia. The treatment of non-ischaemic stenosis is controversial. ${ }^{3}$ Standard coronary angiography with visual assessment and even quantitative coronary angiography on their own, often cannot accurately predict which lesions are causing ischaemia; they may therefore over or underestimate the severity and hemodynamic significance of lesions. ${ }^{4}$ Non-invasive stress and physiological testing, including stress echocardiography and myocardial perfusion imaging are usually robust for detecting ischaemia, but at times, they poorly localise ischaemia to specific vessels and coronary lesions especially in the setting of multi-vessel coronary artery disease. ${ }^{5}$ Invasive fractional flow reserve (FFR) measurements have now been established as a useful physiological adjunct to the anatomical assessment of coronary stenosis during invasive coronary angiography. It has emerged as the gold standard test to assess the haemodynamic significance of coronary lesions. ${ }^{6}$ It typically represents the ratio of maximal myocardial blood flow through a stenosis to the flow in the hypothetical scenario that the artery was normal. The Fractional Flow Reserve Versus Angiography for Multivessel Evaluation trial demonstrated the advantages of FFRguided percutaneous intervention over angiography-only strategy with a significant reduction in major adverse cardiac events in the FFR arm. Despite this, the technique is still not universally applied due to a number of reasons: it adds a further step to the invasive catheterisation procedure and the adenosine infusion required to achieve maximal hyperaemia is poorly tolerated by some patients and can cause considerable discomfort.

\section{Coronary computed tomography angiography (CTA) and CT-FFR assessment}

Multi-slice coronary computed tomography angiography (CTA) was developed in the early 1990's and is being increasingly used to noninvasively identify and exclude anatomically obstructive CAD as an alternative to invasive coronary angiography. ${ }^{7}$ It has a high negative predictive value and is of particularly good use in low-risk populations. ${ }^{8}$ Its limitations however include a high false positive rate, low image quality in some cases and the inability to assess the haemodynamic significance of coronary lesion, especially in moderate or intermediate disease. Previous studies on CTA have shown a tendency towards overestimation of the severity of coronary lesions with only a minority of the high-grade stenosis, as detected by this modality, actually causing ischaemia..${ }^{9}{ }^{10} \mathrm{CT}$-FFR, using computational fluid dynamics and image-based modelling, has thus been developed as a novel and exciting method to establish whether any given stenosis is functionally significant. ${ }^{11}$ Lumped parameter models of the heart, systemic circulation, and coronary microcirculation are coupled to a patient-specific model of the aortic root and epicardial coronary arteries extracted from CTCA data. ${ }^{12}$ Boundary conditions are also set for rest and hyperaemic conditions. ${ }^{13}$ Under resting conditions, total coronary flow is proportional to myocardial mass, $Q c \propto M$ $\beta$ myo (mass can be calculated from myocardial volume, a value obtainable from volumetric CTA data). ${ }^{14}$ According to Murray's Law, the blood flow-vessel diameter relationship can be expressed as $Q \propto d k,(\mathrm{Q}=$ flow rate through a blood vessel, $\mathrm{d}=$ =vessel diameter, and $\mathrm{k}=$ empirically derived constant). ${ }^{15} \mathrm{~A}$ discrete model of the ascending aorta and epicardial coronary arteries is generated and the definition of the boundary conditions for rest and hyperaemic conditions is set. The CT-FFR is then determined by solving the equations of blood flow for the velocity and pressure fields and the CT-FFR is obtained by normalizing the mean hyperaemic pressure field by the average mean hyperaemic pressure in the aorta. Colour contours provide data on the distribution of CT-FFR throughout the coronary tree and numerical values can be obtained at any location. All the data required to compute the result is obtained from the acquired CCTA scans in a single setting at rest and without any modification of CCTA protocols, additional image acquisition or administration of medications. Importantly this helps reduce patient radiation exposure. The efficacy of CT-FFR has been demonstrated in a number of studies. In the Diagnosis of Ischemia-Causing Stenosis Obtained Via Non-invasive Fractional Flow Reserve trial, non-invasive CT-FFR compared favourably with invasive FFR (sensitivity: $87.9 \%$, specificity: $82.2 \%$, 
positive predictive value: $73.9 \%$, negative predictive value: $92.2 \%$ for CT-FFR). The performance of CT-FFRCTA was superior to CTA for diagnosing ischemic lesions. ${ }^{16}$ In the Heart Flow NXT trial, CT-FFR data closely matched those of conventional invasive $\mathrm{FFR}^{17}$ whilst the Determination of Fractional Flow Reserve by Anatomic Computed Tomographic Angiography trial indicated that CT- FFR was more accurate and with better discrimination than $\mathrm{CT}$ alone. ${ }^{18}$

\section{Conclusion}

Calculations of FFR-CT relies on assumptions in the physiological models including patient-specific data and the results are dependent on accurate anatomical models that can be hampered by artefacts from calcification or motion during CTCA image acquisitions. The lengthy turn-around time for data analysis and interpretation currently limits this technology for routine assessment of patients presenting with chest pain. It is likely that these issues will be addressed in a very short space of time allowing this technology to become the new gold standard for non-invasive assessment of CAD.

\section{Acknowledgements}

None.

\section{Conflict of interest}

Author declares that there is no conflict of interest.

\section{References}

1. Ibrahim M, Ussen B, Pottle A, et al. Low-density lipoprotein apheresis is effective in reducing lipoprotein(a) levels and in improving symptoms in a patient with refractory angina secondary to accelerated coronary artery disease. J Clin Lipidol. 2012;6(2):192-194.

2. Lindner JR. The Physiologic Evaluation of Stenosis by Transthoracic Doppler: A Bit of Theory, a Lot of Practice. J Am Soc Echocardiogr 2011;24(4):382-384.

3. Tonino PA, Fearon WF, De Bruyne B, et al. Angiographic Versus Functional Severity of Coronary Artery Stenosis in the FAME Study:Fractional Flow Reserve Versus Angiography in Multivessel Evaluation. J Am Coll Cardiol. 2010;55(25):2816-2821.

4. Fischer JJ, Samady H, McPherson JA, et al. Comparison between visual assessment and quantitative angiography versus fractional flow reserve for native coronary narrowing's of moderate severity. Am J Cardiol. 2002;90(3):210-215.

5. Lima RS, Watson DD, Goode AR, et al. Incremental value of combined perfusion and function over perfusion alone by gated SPECT myocardial perfusion imaging for detection of severe three-vessel coronary artery disease. J Am Coll Cardiol. 2003;42(1):64-70.

6. Pijls NH, De Bruyne B, Peels K, et al. Measurement of fractional flow reserve to assess the functional severity of coronary-artery stenosis. N Engl J Med. 1996;334(26):1703-1708.
7. Tonino PA, Fearon WF, De Bruyne B, et al. Angiographic versus functional severity of coronary artery stenosis in the FAME study fractional flow reserve versus angiography in multivessel evaluation. $J$ Am Coll Cardiol. 2010;55(25):2816-2821.

8. Goldstein JA, Gallagher MJ, O’Neill WW, et al. A randomized controlled trial of multi-slice coronary computed tomography for evaluation of acute chest pain. J Am Coll Cardiol. 2007;49(8):863-871.

9. Budoff MJ, Dowe D, Jollis JG, et al. Diagnostic performance of 64-multidetector row coronary computed tomographic angiography for evaluation of coronary artery stenosis in individuals without known coronary artery disease: results from the prospective multicentre ACCURACY (Assessment by Coronary Computed Tomographic Angiography of Individuals Undergoing Invasive Coronary Angiography) trial. J Am Coll Cardiol. 2008;52(21):1724-1732.

10. Meijboom WB, Van Mieghem CA, van Pelt N, et al. Comprehensive assessment of coronary artery stenoses: computed tomography coronary angiography versus conventional coronary angiography and correlation with fractional flow reserve in patients with stable angina. $J$ Am Coll Cardiol. 2008;52(8):636-643.

11. Kim HJ, Vignon-Clementel IE, Coogan JS, et al. Patient-Specific Modeling of Blood Flow and Pressure in Human Coronary Arteries. Ann Biomed Eng. 2010;38(10):3195-3209.

12. Hirohata K, Kano A, Goryu A, et al. A novel CT-FFR method for the coronary artery based on 4D CT image analysis and structural and fluid analysis. Medical Imaging. 2015.

13. Taylor CA, Fonte TA, Min JK. Computational Fluid Dynamics Applied to Cardiac Computed Tomography for Noninvasive Quantification of Fractional Flow Reserve: Scientific Basis. J Am Coll Cardiol. 2013;61(22):2233-2241

14. Choy JS, Kassab GS. Scaling of myocardial mass to flow and morphometry of coronary arteries. Appl Physiol. 2008;104(5):1281-1286.

15. Murray CD. The physiological principle of minimum work: I. The vascular system and the cost of blood volume. Proc Natl Acad Sci US A. 1926;12(3):207-214.

16. Koo BK, Erglis A, Doh JH, et al. Diagnosis of Ischemia-Causing Coronary Stenoses by Noninvasive Fractional Flow Reserve Computed From Coronary Computed Tomographic Angiograms Results From the Prospective Multicentre DISCOVER-FLOW (Diagnosis of IschemiaCausing Stenoses Obtained Via Noninvasive Fractional Flow Reserve) Study. J Am Coll Cardiol. 2011;58(19):1989-1997.

17. Nørgaard BL, Leipsic J, Gaur S, et al. Diagnostic performance of noninvasive fractional flow reserve derived from coronary CT angiography in suspected coronary artery disease: The NXT trial. J Am Coll Cardiol. 2014;63(12):1145-1155.

18. Min JK, Leipsic J, Pencina MJ, et al. Diagnostic accuracy of Fractional Flow Reserve From Anatomic CT Angiography. JAMA. 2012;308(12):1237-1245. 